\title{
Correspondence
}

\section{MONITORING CHILDREN TREATED WITH CARBAMAZEPINE}

The question of monitoring patients taking anticonvulsants has been reviewed previously. 1,2 During carbamazepine monotherapy, complete blood counts (CBC) and alanine transaminase (ALT) were measured at intervals in 23 children attending a Pediatric Neurology clinic during 1990 and 1991. The results were reviewed retrospectively. The mean age was 10.3 years (SD 3.7 yrs). Prior to starting carbamazepine CBC and ALT were measured in 19 and 14 respectively. In two, high white cell counts (WCC) on the day of a seizure, were excluded from the figures. Maintenance doses were mean $13.09 \mathrm{mg} / \mathrm{kg} /$ day (SD 4.68). The patients were followed for a mean 15.6 months (SD 6.7). The results were divided into those obtained during the first 8 weeks of treatment (22 samples) and those after 8 weeks (62 samples). Drug levels showed compliance in all except for two late in their course of treatment.

Two patients had drug reactions. A 14.5-year-old girl developed petechiae with thrombocytopenia (platelet count $28 \times$ $10^{9} / 1$ ) at 13 days. A 12-year-old girl developed a hypersensitivity reaction at four weeks. Three developed leukopenia. A 15-yearold boy had leukopenia (WCC $<4 \times 10^{9} / 1$ ) from 4 weeks to 8 months. He had a transient neutropenia $\left(1.3 \times 10^{9} / 1\right)$ at 8 weeks. $\mathrm{He}$ was asymptomatic, his dose was not changed and the leukopenia resolved. Another 15-year-old boy had a transient neutropenia $\left(1.4 \times 10^{9} / 1\right)$ at 2 weeks. He also had transient leukopenia on two occasions at 2 weeks and 10 months. A 15 year-old girl had a transient leukopenia at 9 weeks. Two patients had transient elevations of ALT, 109 and $94 \mu / /$ (normal 4-30 $\mu / 1$ ) which on repeat were normal. No symptoms of infection were reported. Another had elevation in ALT (59-65 $\mu / 1)$ over a period of a month associated with a chest infection. Paired t-tests were performed on all $\mathrm{CBC}$ s comparing before and during the first 8 weeks treatment ( 12 patients), and before and after 8 weeks treatment ( 16 patients). There was no significant decrease $(\mathrm{P}<0.05)$ in WCC, lymphocytes, granulocytes nor hemoglobin $(\mathrm{Hb})$. Platelet counts did show a significant decrease $(\mathrm{P}<0.05)$ both during first 8 weeks and after 8 weeks. No platelet count fell below the normal range.

The incidence of hematological abnormalities with carbamazepine has been reviewed. ${ }^{3.4}$ The reported incidence of aplastic anaemia is $5.1 /$ million, agranulocytosis $1.4 /$ million and leukopenia $10 \% .{ }^{4}$ It is not clear whether sequential monitoring can identify patients at risk for the rare serious reactions. Guidelines from the ad hoc committee for the Canadian Association for Child Neurology, regarding blood monitoring during anticonvulsant therapy, suggest performing base line CBC and liver function tests, warning patients of reactions and testing only if symptomatic.' A reply to these guidelines suggested testing young patients treated with valproic acid. Another author also recommended base line blood work and no further monitoring except in high risk groups. ${ }^{2}$ In the present 23 children two clinical reactions occurred prior to routine monitoring and abnormal results did bring 5 back for further testing. Laboratory results did not alter treatment in any. Therefore in this small group of patients treated with carbamazepine and guidelines above (1) would have been appropriate.

Colleen Adams

Philadelphia, U.S.A.

1. Camfield P, Camfield C, Dooley J, et al. Routine Screening of Blood and Urine for severe Anticonvulsant Reactions in Asymptomatic Patients is of Doubtful Value. Can J Neurol Sci 1989; 16: 361-364.

2. Pellock JM, Willmore LJ. A rational guide to routine blood monitoring in patients receiving antiepileptic drugs. Neurology 1991; 41: $961-964$.

3. Hart RG, Easton JD. Carbamazepine and Hematological Monitoring. Ann Neurol 1982; 11:309-312.

4. Pellock JM. Carbamazepine Side Effects in Children and Adults. Epilepsia 1987; 28 (Suppl 3): S64-S70.

\section{RE: CURRENT STATUS OF RADIOSURGERY FOR ARTERIOVENOUS MALFORMATIONS}

To the Editor:

We were interested in the scientific and economic ramifications of the "review article" by Schwartz and colleagues entitled, "Current status of radiosurgery for arteriovenous malformations", published in the Canadian Journal of Neurological Sciences 1991; 18: 499-502. This "review article", by a group that had treated only 18 AVM's at the time of publication of that report, seems somewhat premature especially since many strong conclusions are reached. There are many statements and conclusions stated in this paper that we would like to address.

First, the authors state that two competing technologies exist for the delivery of focused photon irradiation. We would like to emphasize that although a competition might exist between the manufacturers of various devices, there should exist no scientific competition. The International Stereotactic Radiosurgery Society was formed to amalgamate the experiences of neurosurgeons, radiation oncologists, and medical physicists using various techniques of focused irradiation and to share and combine results. As a result one should not feel threatened by other technologies.

Second, Schwartz et al. described at length the concept of radiosurgical accuracy using their modified linear accelerator system. They state that the treatment error vector for antiographically selected targets has a value of $0.3 \mathrm{~mm}$ and that CT scanning is even less accurate than angiography. The gamma unit with a fixed radiation delivery system has a radiation delivery accuracy less than $0.3 \mathrm{~mm}$, and accuracy is significantly dependent upon the neurosurgeons skill at interpreting the stereotactic images as well as target selection. A recent paper by Woo et al. from their linear accelerator center ${ }^{1}$ now reports a clinically observable discrepancy of $4 \mathrm{~mm}$ between the mechanical and radiation isocenters using their system. They have identified the cause of this discrepancy to be the gantry head sag of their linear accelerator and have made recommendations for their device to help correct this. It seems that the physical problems associated with some linear accelerator systems are still being worked out, and that institutional quality assurance must be paramount. 